\title{
Off-the-shelf CAR-T therapy induces remission in child with ALL
}

The impressive response seen in the first leukemia patient treated using donor T cells genetically modified to target the CD19 antigen on tumor cells may signal the opening of a new chapter in the race to commercialize $T$ cell-based chimeric antigen receptor (CAR-T) technologies. A formal clinical trial has yet to commence using the treatment, Paris-based Cellectis's UCARTCD19 procedure. But the initial results, announced in November and presented in December at the American Society of Hematology (ASH) Annual Meeting, suggest that an off-the-shelf formulation of CAR-T cells could challenge the autologous formulations that have already shown striking clinical effects.

Allogeneic cell therapies are generally considered the ultimate product formulation. As off-the-shelf products, they can be shipped in a day or two to patients worldwide: market access is an important consideration for any company. In contrast, a single batch of autologous cells prepared from an individual's own tissue takes weeks to collect and process, with a much greater cost associated with it. Cellectis starts with a commercially available pack of leukapheresis-derived white blood cells collected from healthy donors. From each pack, the company generates hundreds of doses of its product-a number CEO André Choulika expects will ramp up into the many thousands by the time UCARTCD19 is ready for market.

Medical reasons may also tip the needle towards the allogeneic option. This was the case for the 11-month-old girl with relapsed acute lymphocytic leukemia (ALL) who received the experimental formulation of UCARTCD19 cells at Great Ormond Street Hospital in June 2015. A patient may not have enough T cells or cells of high enough quality to make an autologous product, especially after having been exposed to chemotherapy and the ravages of their disease. Current clinical studies involving the National Cancer Institute and The University of Texas MD Anderson Medical Center in Houston are using CAR-T CD19 cells derived from matched donors to address these concerns. But although allogeneic, they are not off-the-shelf formulations. The urgency to reduce tumor size immediately may also rule out an autologous treatment. "An off-the-shelf product may be given as a first approach," Choulika says. "At least you have a vial you can pull out of the freezer, inject into the patient, and have an interesting clinical benefit."

When the request for compassionate use came in from the London-based children's hospital, Cellectis had already manufactured and quality-tested a bank of donor cells in preparation for its phase 1 trials. In addition to using its gene editing tool-transcription activator-like effector nucleases (TALENs) - to give the chimeric cells the ability to target CD19, the French biotech used TALENs to disrupt T-cell receptor expression and in so doing overcome human leukocyte antigen barriers and address the risk of graft-versus-host disease. It also targeted CD52 with TALENs, to render the engineered cells resistant to the leukemia drug Campath (alemtuzumab) made by Sanofi-owned Genzyme, used as a preconditioning treatment, though Choulika confirms that the final product will not include CD52 engineering. The treatment induced a remission in the London-based child that was still in effect five months later, in December, when the announcement at the ASH was made.

Of course, data from one patient under compassionate use do not come close to equaling the proof of concept already seen with autologous CAR-T cells. Two years ago at ASH, researchers from the University of Pennsylvania in Philadelphia, in partnership with Basel-based Novartis, showed that in initial trials in ALL, five of five adults and 19 of 22 children treated with an autologous CD19-targeting CAR-T experienced a complete response. The data spawned a host of partnerships around both allogeneic and autologous CAR-T (Nat. Biotechnol. 33, 571, 2015).

And alliances

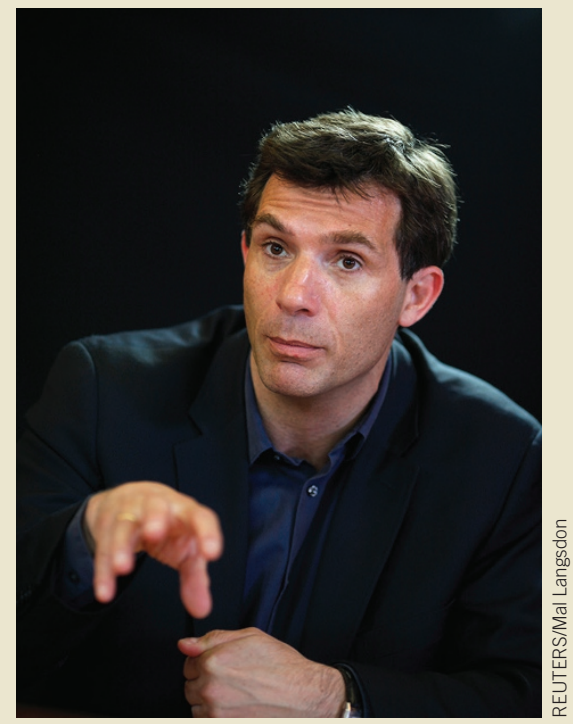

Andre Choulika, CEO of Cellectis continue to flourish. On November 19, Suresnes, France-based Servier exercised its option to worldwide rights to UCARTCD19, simultaneously announcing it was bringing in New York-based Pfizer as a commercialization partner for the product. Fewer than two weeks later, Adaptimmune, in Abingdon, UK, and Seattle startup Universal Cells announced that they would be joining forces to produce off-the-shelf TCR-engineered T-cell products for cancer immunotherapy. The collaboration combines Universal Cells' nuclease-free technology, which centers on the use of a stem cell-tropic recombinant adenoviral vector (AAV) serotype for engineering human pluripotent stem cells, with Adaptimmune's increased affinity T-cell receptor technology. But CEO Claudia Mitchell points out that Universal Cells is years from the clinic. "We still have to develop the ideal protocol to get from iPS [induced pluripotent stem] cells to T cells," she says.

Also actively publishing in the allogeneic CAR-T race is Sangamo Biosciences of Richmond, California. The zinc finger protein-oriented genome editing specialist is considering partnerships to allow them to enter the cancer immunotherapy field. "Everyone who has a [genome-edited based] targeting technology currently uses either a transposon approach to inserting a chimeric antigen receptor or a lentivirus approach," says executive vice president, research and development, Geoff Nichol. "Neither are ideal manufacturing approaches." Insertion using lentivirus-the delivery method Cellectis uses in UCARTCD19-is not very robust and may be the subject of intellectual property challenges. "Sangamo is developing an alternative manufacturing approach that would allow us to insert a targeting moiety," Nichol says, using a combination of zinc finger nuclease mRNA and AAV delivery. Choulika acknowledges the limitations of lentiviral vectors. "They are usually developed to infect quiescent cells," he says. But T cells divide. "You can use different kinds of vectors," he says. "I don't know yet where we will stand at the time of the commercialization," he says, either with respect to switching to another vector type or securing a license to use lentiviral delivery.

Mark Ratner Boca Raton, Florida 